\title{
BASIC THINKING ABOUT BÁSIC SCIENCE - A PHENOMENAL APPROACH
}

\author{
Nagalakshmi A. Rao*
}

\begin{abstract}
Traditionally, science teaching comprises of theory lectures coupled with conventional laboratory sessions. In recent years, one observes a downslide and declining interest in basic science education among the student community. Numerous reasons are attributed to this malady. With the advent of computers, supplemented by softwares like Mathematica, Matlab, etc., physics education can be made more exciting, enriching and challenging. Computational Physics will enhance the science culfure and is a promising opproach to revive and revitalise the interest of students fowards pure science. Technology enriched hands-on, minds-on laboratory is the need of the hour.
\end{abstract}

\section{Introduction}

At the threshold of the $21^{\text {st }}$ century one observes a downslide and declining interest in basic science education among the student community. Students at the preuniversity level opt professional courses - IITs, Engineering, Medical or Dental and only the less-previleged ones enter basic science. Numerous reasons are attributed to this malady. Among them are:

* Department of Physics, Government Science College, Bangolore. 

a. conventional and overloaded syllabi
b. lack of appropriate method of evaluation
c. reduced job opportunities
d. lack of creative and conducive environment

Science has a culture of its own and internalisation of this culture should be the aim of teaching and learning.

$\begin{array}{ll}\text { Learn how to LEARN } & \text { (Theory) } \rightarrow \text { Teaching } \\ \text { Learn how to DO } & \text { (Practicals) } \rightarrow \text { Learning } \\ \text { Learn how to LIVE } & \text { (Examination) } \rightarrow \text { Evaluation }\end{array}$

As a part of the curriculum, scienctific temper can be spread by conducting Quiz contest and Just-A-Minute Contest, where knowledge is imparted informally. Communication skills are sharpened and the culture of science is disseminated in its true spirit.

Lab work is not only a defining feature of science, but also an essential ingredient in giving students the adequate technical skill. The science curriculum should expose the students to the fascinating world of reality and enable them to:

- observe keenly and carefully

- invoke a spirit of enquiry

- nurture and nourish the creativity and innovativeness.

\section{Teaching Curriculum}

The teaching curriculum in science should be designed so as to foster creativity and stimulate critical thinking. The curriculum should focus on making the students technologically literate and empower them to lead a productive life. The role of workshop practice in science is expected to make the study of science more attractive besides improving the job potential. Some novel techniques that could be employed to make physics education more instructive are :

i. industry-based curriculum

ii. job-oriented vocational courses 
iii. interdisciplinary approach - Pure Physics \& Applied Physics

iv. twinning programmes - basic science with NIT, $\| \mathrm{HT}$, etc

v. offer dual degree programmes

vi. computational physics

Physics is an integral part of the science curriculum. Computer assisted learning plays an important and significant role in illustrating the concepts of physics effectively. It enhances individual and creative thinking. Problem-solving and data analysis are simplified, graphics and animation are illustrative and this is the right step to make science very interesting and exciting.

\section{Role of Computational Physics}

In the IT Sector computers boost the performance, improve the creativity and give a career edge. Interestingly, sharply defined, varied needs for. IT professionals in various industries have emerged. The main objective of computational physics is to train students in:

1. Programming language

2. Numerical methods

3. Develop algorithms and programs for physics problems

Many numerical programming languages are known - BASIC, QBASIC, FORTRAN, PASCAL, C, $\mathrm{C}^{++}$and so on. Recently symbolic languages like Reduce, Mathematica, Maple, MatLab, etc are also available, which serve as powerful tools in numerical computation and graphical illustration. Such programming languages are effective in solving many physical problems like:

i) Roots of quadratic equation

ii) Simple matrix multiplication

iii) Simple harmonic motion

iv) Analysis of LCR circuits

v) Solution of one dimensional Schrodinger equation for various potentials like the square well potential, harmonic oscillator potential, Coulomb potential, etc. 
In general, computer based experiments help the students to study the effects of

a) change of step size

b) change of the initial condition of the physical system

c) change of physical parameters of the system

This helps in better understanding of the underlying physical concept. A course rich in physical content merged with computational analysis gives a deeper insight and is easier to visualise.

\section{Potentiality of Mathematica}

Mathematica, a modern software developed by Stephen Wolfram, serves as a powerful tool in numerical computation and symbolic manipulation. Unlike the traditional languages, Mathematica is unique, because a single command replaces a set of program stotements. This novel programming language can be used for solving mathematical problems of all kinds - starting from the undergraduate level to research oriented ones. Because of its ability to deal with symbolic expressions as well as numerical funcions, Mathematica has the largest computational potential. Within seconds, one can get the value of $\mathrm{Pi}$ to a precision of 100 digits or even more. Mathematica can perform integration, differentiation, matrix multiplication, can solve a wide range of differential equtions, can create graphics and work with formulas of any length - solving problems that would have taken years by hand. On many computers, Mathematica can not only provide static visual images, but also stunning animated graphics.

Mathematica has a special built-in library with more than 1500 functions, like Expand, Solve, Integrate, FindRoot, etc., each having its own unique algorithm. Information on any Mathematica funtion can be obtained by entering "?" followed by the command. To obtain the graphical solution, options like Plot, ParametricPlot, Plot3D are used.

\section{Conclusions}

"The whole of science is nothing more than refinement of everyday thinking" - Albert Einstein.

To summarise, a tranformation from the conventional method of teaching, which is based on "passive interaction" to the "active and creative interaction" is the need of the hour. 
Conventional Method:

Prevailing System:
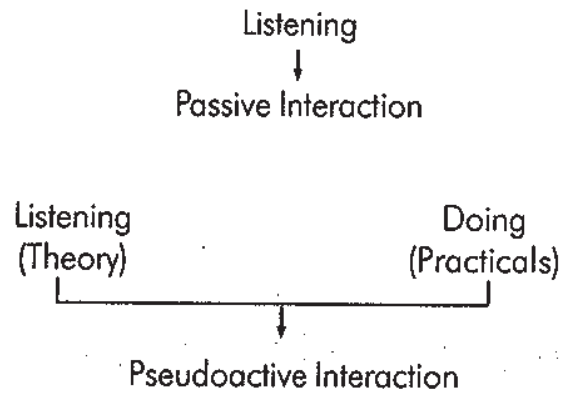

Scientific Approach :

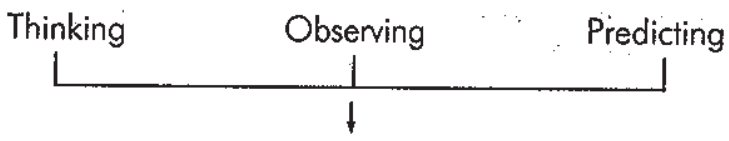

Active and Creative Interaction

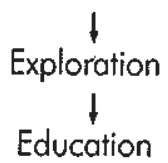

Teaching methodology should be refined, the relevance of lab work emphasised, seminars to be encouraged and scientific temper promulgated. Students should be encouraged to fabricate simple innovative, low-cost, concept-centered experiments. Students should be motivated to launch "Wealth from Waste" projects, where discarded materials are put to use. 